\title{
CORRIGENDUM
}

\section{Family-based association study of DTNBP1 in 6p22.3 and schizophrenia}

JX Tang, J Zhou, JB Fan, XW Li, YY Shi, NF Gu, GY Feng, YL Xing, JG Shi and L He

Molecular Psychiatry (2003) 8, 1008. doi:10.1038/sj.mp.4001430

Correction to: Molecular Psychiatry (2003) 8, 117-118. doi:10.1038/sj.mp.4001287

Following publication of the above paper, the authors have identified an error in Table 1. The correct version of the Table 1 is reproduced below.

Table 1 Estimated haplotype probabilities and chi-squared test of mulimarker haplotypes using TRANSMIT

Marker (NCBI Number ${ }^{a}$

P1328 (rs742105)

P1655 (rs2619539)

P1763 (rs2619522)

P1578 (rs1018381)

P1583 (rs909706)

Estimated probability

$\mathrm{O}^{\mathrm{b}}$

$\mathrm{E}^{\mathrm{c}}$

$\operatorname{Var}(\mathrm{O}-\mathrm{E})^{\mathrm{d}}$

$\chi^{2}$ (1 df)

$P$-value

Global $P$-value
Haplotype

$\begin{array}{ccccc}\mathrm{T} & \mathrm{C} & \mathrm{T} & \mathrm{C} & \mathrm{C} \\ \mathrm{G} & \mathrm{G} & \mathrm{C} & \mathrm{C} & \mathrm{C} \\ \mathrm{T} & \mathrm{T} & \mathrm{T} & \mathrm{T} & \mathrm{G} \\ \mathrm{C} & \mathrm{C} & \mathrm{C} & \mathrm{C} & \mathrm{T} \\ \mathrm{A} & \mathrm{A} & \mathrm{G} & \mathrm{G} & \mathrm{G} \\ 0.37 & 0.26 & 0.12 & 0.1 & 0.05 \\ 183.83 & 115.31 & 60.16 & 41.67 & 22.08 \\ 166.69 & 116.46 & 55.57 & 46.72 & 22.89 \\ 47.17 & 42.84 & 23.45 & 18.37 & 11.04 \\ 6.22 & 0.03 & 0.90 & 1.39 & 0.06 \\ 0.01 & 0.86 & 0.34 & 0.24 & 0.81 \\ 0.00072^{\mathrm{e}} & & & & \end{array}$

